\title{
Interest of Biodentine as Pulp-Capping Materiel under CAD/CAM
}

\section{Ceramic Inlay}

\author{
Meriem Ben Miled ${ }^{1}$, Rihab Dakhli ${ }^{2 *}$, Mohsen Maaned ${ }^{1}$, Hayet Hajjami ${ }^{3}$, Mounir Cherif ${ }^{3}$
}

\author{
${ }^{1}$ Resident, Department of Fixed Prosthodontics, Dental Faculty of Monastir, University of Monastir, Tunisia \\ ${ }^{2}$ Associate Professor, Research Laboratory of Occlusodontics and Ceramic Prosthesis LR16ES15, Faculty of Dental Medicine, \\ University of Monastir, Monastir, Tunisia \\ ${ }^{3}$ Professor, Department of Fixed Prosthodontics, Faculty of Dental Medicine, University of Monastir, Monastir, Tunisia
}

DOI: $10.36347 /$ sjmcr.2021.v09i03.003 $\quad$ | Received: 18.02.2021 | Accepted: 02.03.2021 | Published: 04.03 .2021

*Corresponding author: Rihab Dakhli

Abstract

Recently, tissue economy is considered as one of today's key preoccupations. The preservation of pulp vitality falls within this context and must be attempted, especially in the case where the tooth is intended to serve as abutments of fixed restauration. For this reason, direct pulp capping, previously contraindicated under a fixed prosthesis, is now recommended and possible thanks to the recent appearance on the market of new biomaterials, in particular Biodentine. It is a bioactive cement with dentin-like mechanical properties that allows it to withstand contraction forces underneath direct or indirect restorations.

Keywords: Pulp-Capping, Ceramic, Inlay, Biodentine.

Copyright $\left({ }_{0} 2021\right.$ The Author(s): This is an open-access article distributed under the terms of the Creative Commons Attribution 4.0 International License (CC BY-NC 4.0) which permits unrestricted use, distribution, and reproduction in any medium for non-commercial use provided the original author and source are credited.

\section{INTRODUCTION}

Nowadays, it is indisputable that the notion of tissue economy guides the practitioner's steps in his daily practice. This is why, when faced with a deep carious lesion in a tooth that is a candidate for a prosthetic restoration, endodontic treatment is left as a last resort. In fact, several studies have shown that the loss of pulp vitality is one of the most important biological causes of prosthetic failure. Therefore, researchers have been investigating practical solutions for the preservation of pulp vitality. Several pulp capping products such as Calcium hydroxide, MTA and Biodentine have been developed.

For many years, calcium hydroxide has represented the "gold standard" for direct or indirect pulp capping cases. Nevertheless, it has been shown that this material has several disadvantages, such as its low mechanical strength, its low adhesive potential to dentin and, above all, its aggressive effect on the pulp tissue.

Most recently, a calcium-silicate based cement (Biodentine $^{\mathrm{TM}}$, Septodont) has been proposed, used in various clinical applications, such as root perforations, apexification, resorptions, retrograde fillings, pulp capping procedures, and dentine replacement. This material presents high compressive strength and is biocompatible. In fact, it induces the production of pulp fibroblastic protein at the contact of the pulp. In addition, it is a bioactive bio-mineralizing material that allows the production of restorative dentine and, consequently, an almost perfect seal to the dentine [1, 2].

In this clinical report, we present a case of a patient with a previous history of pulp complications after restoration of deep caries with conventional protocols.

\section{Case Report}

A 33 years old female patient reported to our department with the complaint of pain in the first upper left molar recently restored with composite resin. Initially, the tooth were tested positive on pulp vitality and negative on percussion. Radiographic evaluation revealed that there was a critical proximity between the composite restoration and pulp (Figure-1). The patient was informed about the need of removing the composite filling and the possibility that an endodontic treatment would be required. 


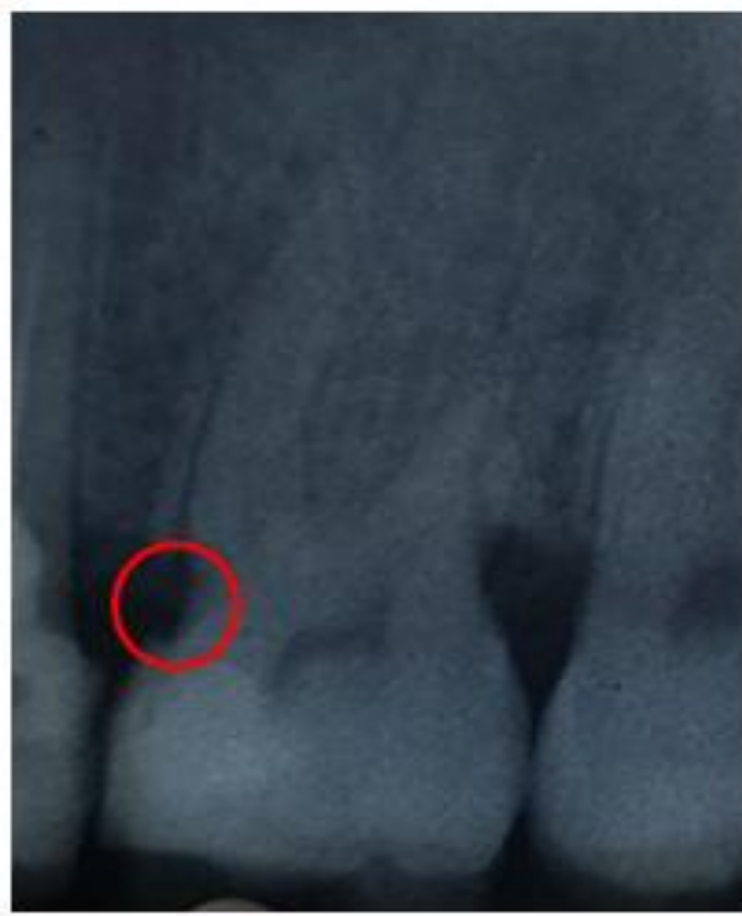

Fig-1: Radiograph before the composite removal

After patient consent, local anesthesia was performed and a rubber dam was placed to avoid contamination with saliva. The removal of composite restoration provoked an iatrogenic pulp exposure (Figure-2). Clinically the pulp of the tooth at the exposure site was vital without any major bleeding, so we have opted for the maintenance of tooth vitality by direct pulp capping.

The control of the hemorrhage was achieved by pressing moist cotton pellets on the pulp wound for 1-2 min and then we have disinfected the cavity with $3 \%$ sodium hypochlorite and gently dried the surrounding dentin it a two-way syringe.

Biodentine $^{\mathrm{TM}}$ (Septodont) was chosen as a pulp capping and provisional filling material. It was placed as a bulk material to restore the entire cavity (Figure-3). The material was left in place for 6 weeks.



Fig-2: Pulp exposure

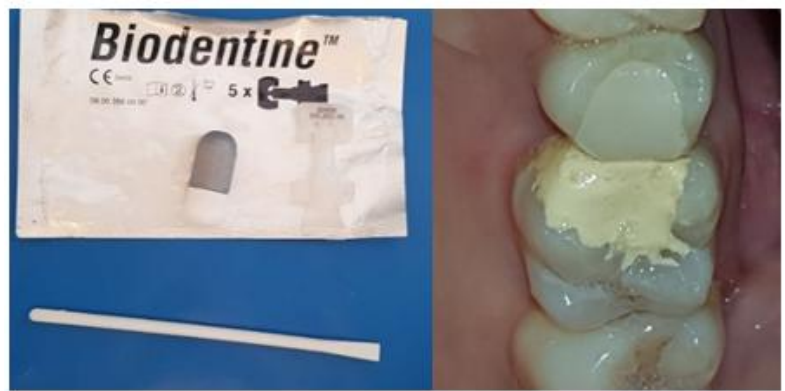

Fig-3: Biodentine placed as a bulk material to restore temporarily the tooth exposure

The patient reported that the tooth remained symptom free during the 6 weeks period. Nevertheless, she was tested positive to the cold spray test. The biodentine filling was reduced and kept as dentin substitute (Figure-4). Then, the tooth was prepared for receiving an hybrid ceramic inlay and a final impression was taken using the one-step putty-wash technique.



Fig-4: Biodentine ${ }^{\mathrm{TM}}$ was partially removed to serve as a dentin substitute and the tooth was prepared for receiving a hybride ceramic inlay

Following the conception and fabrication of the inlay onlay with CAD/CAM and its validation, we moved to the final step, which is prosthesis's bonding.

Selective enamel etching was done for 15 seconds after having covered the whole cavity enamel contour and dentin with orthophosphoric acid $37 \%$ to improve the effect of the one-step self-etching system followed by primer application (Figure-5).
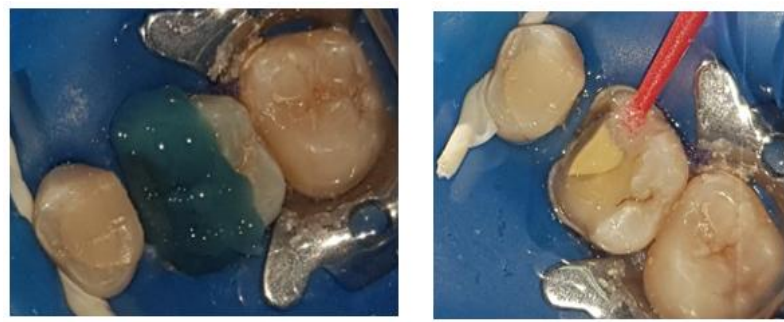

Fig-5: Treatment of the dental surface: a-Selective enamel etching done for 15 seconds followed by hole surface etching for 15 seconds, $b$ : Self-etching primer application The same bonding procedures were carried out at the same time on the restoration. Hydrofluoric acid 
9\% is applied on the underside surface of the prosthesis and then rinsed with water and air-dried. Silane is applied to the restoration and to the composite build-up for one minute and then completely air-dried (Fig-6).



Fig-6: Application of the silane on the underside surface of the restoration with a microbrush after etching

The dual cure resin cement was applied on the restoration the restoration is then placed into the cavity and firmly pushed allowing the excess material to flow out of the cavity. We can use a brush to remove the little bit of composite cement from the restoration-tooth interface. Initial polymerization is done under mild pressure with an instrument. Another polymerization cycle is carried out under glycerin to isolate completely from oxygen that might inhibit curing of the outer layer. Once the rubber dam is removed, occlusion is checked and final surface polishing is performed (Figure-7).

One week after Bonding, the patient was reexamined and reported no postoperative sensitivity.

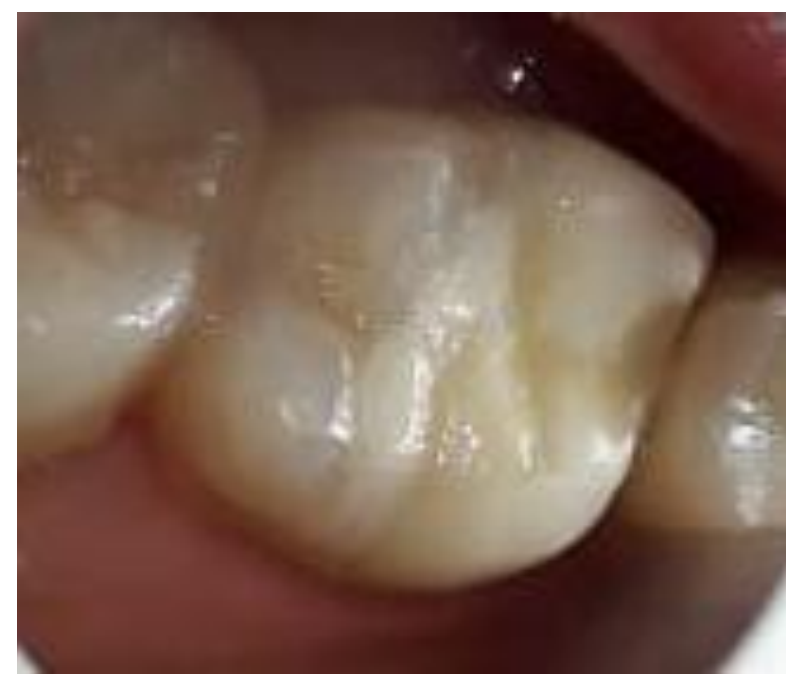

Fig-7: Definitive restoration after checking the occlusion DISCUSSION
When it is about indicating an indirect restoration on a vital teeth with extensive carious lesion and before the beginning of the prosthetic treatment it is necessary to check out the depth and its pulp proximity. In this case, the removal of the composite restoration led to a pulpal exposure. It was imperative to choose a material that not only protects the exposed pulp and replaces lost dentin, but also serves as a solid base for the final restorative material. That is why we have chosen Biodentine. In fact, it is a new generation of silicate cement, which is as bioactive as mineral trioxide (MTA), but with superior handling properties and strength [3].

In fact, histological studies have shown that biodentine affected favorably healing when placed directly in contact with the pulp by enhancing the proliferation, migration, and adhesion of human dental pulp stem cells. This confirms the bioactive and biocompatible characteristics of the material. Its ability to trigger reparative dentin formation together with its antibacterial properties ensure long-term preservation of pulp vitality that is highly required in restorative dentistry $[4,5]$.

In addition, Compared to conventionally used pulp capping materials, such as calcium hydroxide and MTA, Biodentine showed the highest compressive strength. In fact, the product sheet of Biodentine states that a specific feature of Biodentine is its capacity to continue improving in terms of compressive strength with time until reaching a similar range with natural dentine (elastic modulus of $22 \mathrm{GPa}$, compressive strength of $220 \mathrm{MPa}$ and micro-hardness of $60 \mathrm{VHN}$ ). This property is highly requested especially in the molar region where restored teeth needs to withstand high masticatory forces [6].

Moreover, Studies carried out on the quality of adhesion of Biodentine to dental tissues have shown that thanks to this material, an almost perfect adaptation with the enamel-dental complex is achieved. This process is continuous over time, thus increasing the tightness of the seal between the biomaterial and the dental tissues.

Indeed, scanning electron microscope imaging has shown, at the entrance of the dentinal tubules, a recrystallization that leads to the formation of mineral tags inducing a micromechanical anchoring of the Biodentine [7].

This fact allows the preservation of the material as a base underneath direct/indirect restoration.

The durability of the reconstruction, apart from the used materials, is influenced by the quality and sufficiency of their bond. The use of Biodentine requires, as the manufacturer suggests, covering it with a final material after the prior use of an adhesive agent. 
For this reason, it is important to evaluate the shear bond strength of Biodentine to a composite material. Studies have shown that Biodentine is a weak restorative material in its early setting phase. Therefore, the placement of the inlay must be delayed for more than 2 weeks, so that Biodentine material will undergo adequate maturation to withstand contraction forces from the resin composite [8].

It is true that ceramics are the most biocompatible materials and give the best esthetic result, but it is important to ensure that the replacement material has the mechanical properties that are closest to the tooth substrate to be replaced. In fact, the closer the modulus of elasticity of the restorative material and the supporting dental tissue are to each other, the more similarly they respond to the stresses developed during functional movements.

For a ceramic layer uniformly supported by and bonded to a less stiff material, high tensile stresses develop in the ceramic at its interface with the cement, directly below the loaded area. These interfacial stresses arise from strain differences in the ceramic, cement, and dentin because of the ceramic being much stiffer (higher elastic modulus) than either the cement or the dentin.

Considering the fact, that the closer we get to the pulp, the more the mechanical properties of the dentin decrease, the stiffness of the ceramic material exposes the material to the risk of fracture. That is why composite or hybrid restorations are preferred over ceramic in cases of loss of dentin substance $[9,10]$.

\section{CONCLUSION}

Biodentine appears to be an effective materiel for the preservation of pulpal vitality and a dentine substitute with good dentine adhesion as well as to the overlying adhesive system. Thanks to all these qualities, the use of this materiel can be considered indispensable for a prosthetic restoration to respect the principle of tissue economy.
Conflict of Interests: The authors declare that there is no conflict of interests regarding the publication of this paper.

\section{REFERENCES}

1. Mishra L, Basu P, Dash S, Singh NR, Behera R. Dentine Replacement with Biodentine Under Class II Composite Inlay: A Case Report. Indian Journal of Forensic Medicine \& Toxicology. 2020 Oct $1 ; 14(4)$.

2. Dammaschke T. A new bioactive cement for direct pulp capping. Int Dent. 2012;7:52-8.

3. Malkondu Ö, Kazandağ MK, Kazazoğlu E. A review on biodentine, a contemporary dentine replacement and repair material. BioMed research international. 2014 Jun 16;2014.

4. Luo Z, Li D, Kohli MR, Yu Q, Kim S, He WX. Effect of Biodentine ${ }^{\mathrm{TM}}$ on the proliferation, migration and adhesion of human dental pulp stem cells. Journal of Dentistry. 2014 Apr 1;42(4):4907.

5. Shayegan A, Jurysta C, Atash R, Petein M, Abbeele AV. Biodentine used as a pulp-capping agent in primary pig teeth. Pediatric dentistry. 2012 Dec 14;34(7):202E-8E.

6. Grech L, Mallia B, Camilleri J. Investigation of the physical properties of tricalcium silicate cement-based root-end filling materials. Dental Materials. 2013 Feb 1;29(2):e20-8.

7. Atmeh AR, Chong EZ, Richard G, Festy F, Watson TF. Dentin-cement interfacial interaction: calcium silicates and polyalkenoates. Journal of dental research. 2012 May;91(5):454-9.

8. Hashem DF, Foxton R, Manoharan A, Watson TF, Banerjee A. The physical characteristics of resin composite-calcium silicate interface as part of a layered/laminate adhesive restoration. Dental Materials. 2014 Mar 1;30(3):343-9.

9. Kelly JR. Clinically relevant approach to failure testing of all-ceramic restorations. The Journal of prosthetic dentistry. 1999 Jun 1;81(6):652-61.

10. Huth KC, Chen HY, Mehl A, Hickel R, Manhart J. Clinical study of indirect composite resin inlays in posterior stress-bearing cavities placed by dental students: results after 4 years. Journal of dentistry. 2011 Jul 1;39(7):478-88. 